\title{
Design of Digital Reading Interface Considering User Experience
}

\author{
Chen Jing ${ }^{1, a}$, Li You, Du Ping ${ }^{1, a}$ \\ ${ }^{1}$ Wuhan technology and business university, Wuhan, 430081, China \\ aemail: chenjing_wht@163.com
}

\begin{abstract}
Keywords: Universal Usability; Distributed Cognition Theory; Effort Model; interactive Design
\end{abstract}
\begin{abstract}
The issue of diversity of public users' cognitive competence is always one big challenge in the interactive design field of digital reading material interface. The author analyzes and models the interactive activities of digital reading material interface based on the Distributed Cognition Theory, confirms the relation between the interactive activities and information in the process of human-computer interaction, and, on the basis, raises the interactive design method of digital reading material interface based on users' cognitive competence. When adopting this method, the designers shall firstly conduct user survey and identity the user groups in need of care, then analyze the cognitive competence, establish the users' effort model, and describe users' interactive behaviors, confirm the basic interactive framework, and establish the interactive design matrix by using the universal usable design method, and raise the interactive design scheme. We take the digital reader terminal as the case, adopts the method to formulate the design scheme, and verify the validity of the design method by comparing the design scheme with the traditional method. The interactive design method can help designers develop easily understandable and easy-to-use digital reading material interface, reduce users' effort models and better satisfy digital reading material users' needs of cognitive diversity.
\end{abstract}

\section{Introduction}

With the popularization of the digital reading materials in every service field, and it's user groups is increasingly widespread; however, the public users have different educational degrees and computer operation experiences. The traditional interactive design method of digital reading material interface are mainly oriented towards the user groups with rich operation experiences in computer products and are not sufficient to meet the public users' needs of cognitive diversity. The paper considers the self-service registration service terminal interface of digital reading materials as the case of interactive design, based on the research of the interactive design modes of digital reading material interface, introduces users' cognition-based interactive design method and design processes of digital reading material interfaces, and presents the design scheme to realize the aim of decreasing users' effort models and meeting their needs of cognitive diversity.

\section{The Establishment of the Interface Interactive Models}

Simplifying the interface interactive processes and providing the interface information easily understood by the public users are two main approaches to reducing users' effort models occurred in the human-computer interaction. Researching the interactive design of digital reading material interface based on Distributed Cognition Theory and Universal Usability Design Method can provide the necessary support for the realization of the aforesaid approaches. Applying the resource modes of the Distributed Cognition Theory into the development process of the digital reading material interface can simplify the interface interactive processes, distribute the cognitive resources to the system, and provide reasonable supplies to help users understand the interface information to reduce users' effort models at the most extent and let the user groups with different cognitive competences be capable of completing the basic interactive tasks. The interface interactive models and design method of digital reading materials based on this can help designers make reasonable decisions of the interface information distribution. 


\section{Interface Information Structure}

Unlike the traditional human-computer interface, the digital reading material interface is mainly characterized by single-window full-screen operation. The users only can complete the specific operation tasks in accordance with the fixed and single interactive processes, which presents higher requirements for the system's procedures. Meanwhile, due to the public use environments of digital reading materials, users are influenced greatly by the contextual factors in the interactive process, so it is feasible to introduce the processes and contextual factors into the resource models and establish the corresponding information interactive strategies and the interface interactive models oriented towards digital reading materials to describe the relations between interactive movements and information resources in order to help the designers analyze whether the proposed design scheme can reduce users' memory selection load and realize the aim of reducing users' effort models. The information structure of the digital reading material interface interactive models is shown in figure1. The processes in Table I is defined as the set of operation sequences. The description of the processes can conduct timely tracking to the targets. Contexts are defined as the status or variables related to users' interactive activities in the process of human-computer interaction.

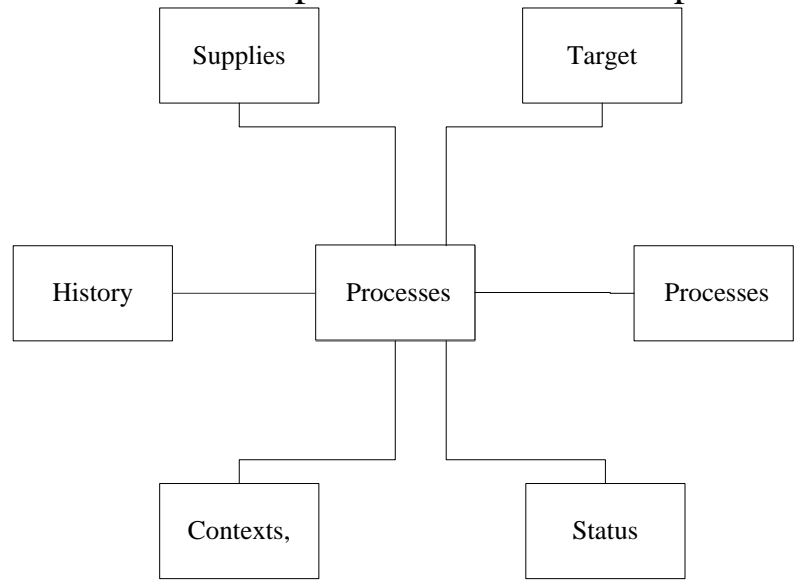

Fig.1. The information structure of interactive model of digital reading material interface

\section{Interface Interactive Strategies}

The interface interactive strategies proposed by the interactive models of digital reading material interface include the following three ones: target matching and optimization, the establishment and evaluation of processes, and the identification of supplies. The three strategies are used to describe the users' possible behaviours in the interactive process and the corresponding operation provided by the interface system. In the interactive process of users and interface systems, these strategies can be used singly or simultaneously or in combination.

(1) Target matching and optimization: the resource model's treatment for targets was the target matching strategy at the most early stage, which can be used in any periods in the process of task execution. The strategy of target matching and optimization in the paper introduces the descriptive interactive movements of the process-contextual factors, and redefines the function of target matching which includes the target breakdown and evaluation based on the processes and contexts. The detailed description is shown in figure 2 . 


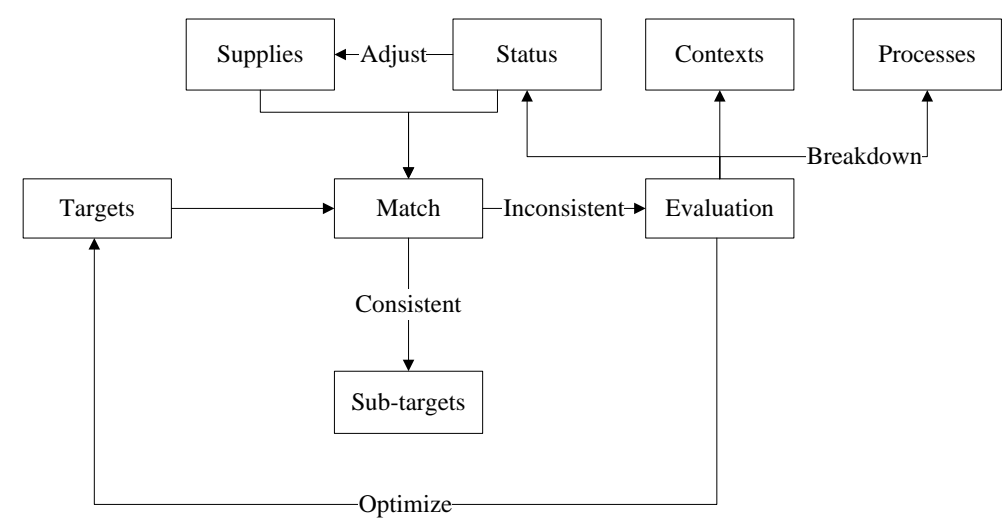

Fig.2.

(2) Establishment and evaluation of processes: in the human-system interactive process, users shall confirm the next interactive movement this is likely to adopt through the information of the current system status and supplies, etc. The set of interactive movements form the basic operation process. The highly-efficient and concise operation process facilitates the aim realization, or otherwise hinders it. The detailed description is shown in figure 3.

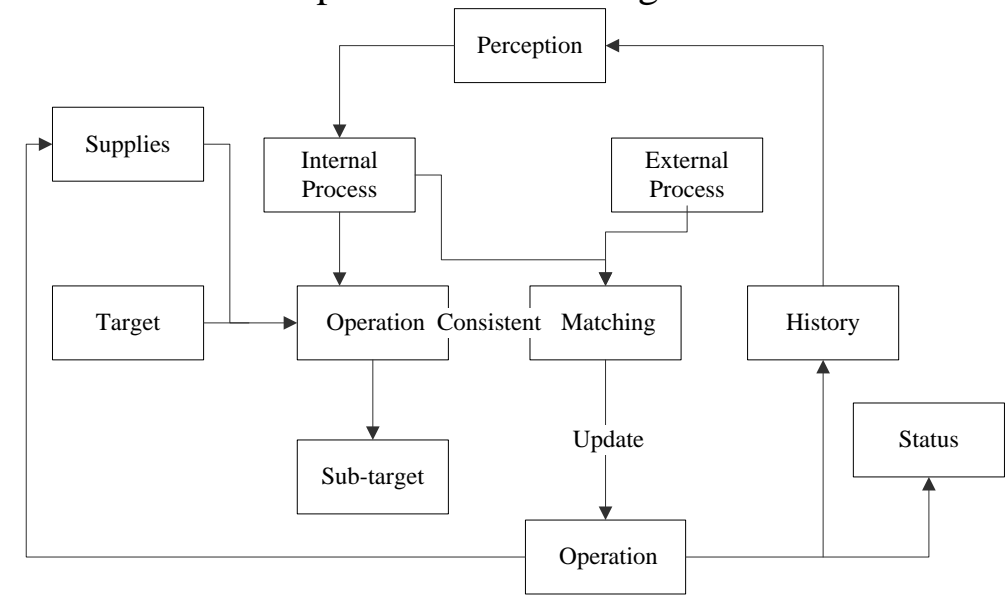

Fig.3. Establishment and evaluation of processes

(3) Identification and Acquisition of Supplies: whether acquiring the correct supply relations or not determines the success of failure of the whole interactive task. In the current system's interactive process, users identify the executable operation by combining their own knowledge, the current situation and system status, realize the transition from the current target to the next target through a series of operations, conduct the assessment in combination of targets, and finally confirm the acquisition of the supplies. The detailed description is shown in Table 4.

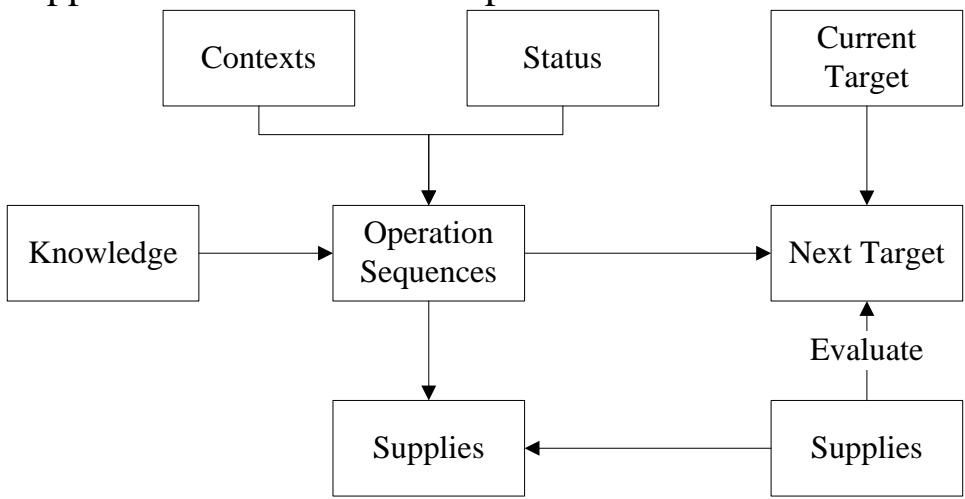

Fig.4. Identification and acquisition of supplies

The information structure and interactive strategies above are sufficient for the rather simple human-computer interaction analysis towards digital reading materials, can provide a coherent core for the interactive modelling of digital reading material interface, and instruct the establishment of design method. 


\section{The Research of Users' Cognition}

\section{The formulation of design scheme.}

In the formulation period of design scheme, designers need design the matrix by referring to the universal usable digital reading material interface of users' effort models, and establish the users' effort model-oriented interactive design scheme and make preparation for the development of the experimental prototype system.

The formation of the detailed interactive design scheme depends on the specific interactive tasks, and the designers also need refer to the interactive design standards of digital reading material interface. In this very stage, the interactive design scheme is mainly shown in the form of design document which shall include the five items of detailed information framework pattern, interface layout, interface factors, task processes and interface sequences.

\section{The framework of prototype system.}

In the stage of system realization, designers need design and realize the concrete interface contents by referring to the design documents after confirming the interactive design scheme. The universal usable matrix and design scheme of digital reading materials can allow users with different cognitive levels to understand the interface information.

In order to realize the complete interactive process and let the users experience the real visual effect, Flash and other interface design tools of digital reading materials can be used.

\section{Assessment of effort models.}

The degrees of effort models are directly connected with the identification and acquisition of supplies. It is discovered from the related researches that the operation performance isn't directly connected with the levels of effort models. Some experimental schemes with high performance usually cannot win users' high subjective satisfaction scores, mainly because users generate high effort models when interacting with the system. Therefore, it is necessary to evaluate users' effort models. Users' effort models in the human-computer interaction behaviors cannot be directly measured but evaluated through related indirect indicators.

The evaluation results are shown in Tables 5-7, which respectively represent users' effort model indicators, satisfaction degrees and operation performances after completing the registration and check-in tasks in the prototype system and reference system. From the statistic results, we can discover that users' effort model indicators, satisfaction degrees and operation performances when operating the three tasks by using the prototype system are better than those from the reference system.

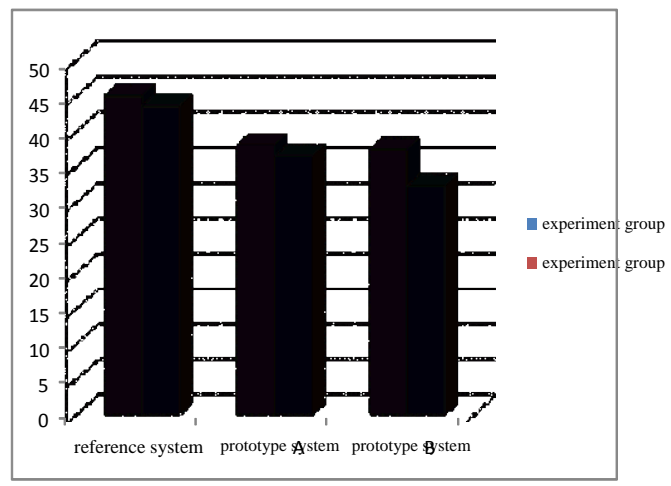

Fig.5. The comparison of the average values of effort model degrees 


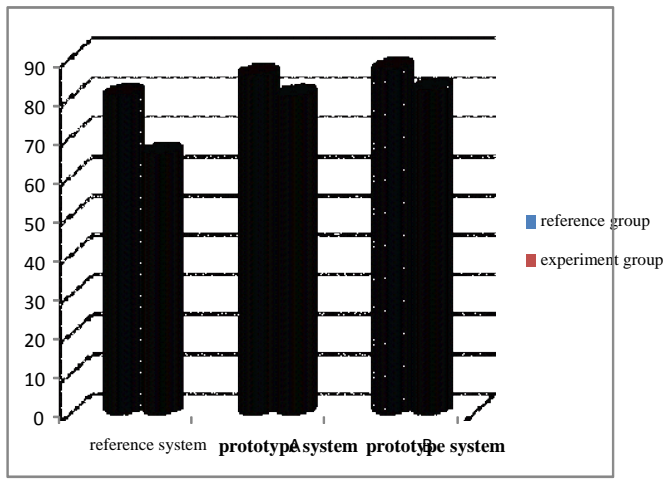

Fig.6. The comparison of the average values of system satisfaction degrees

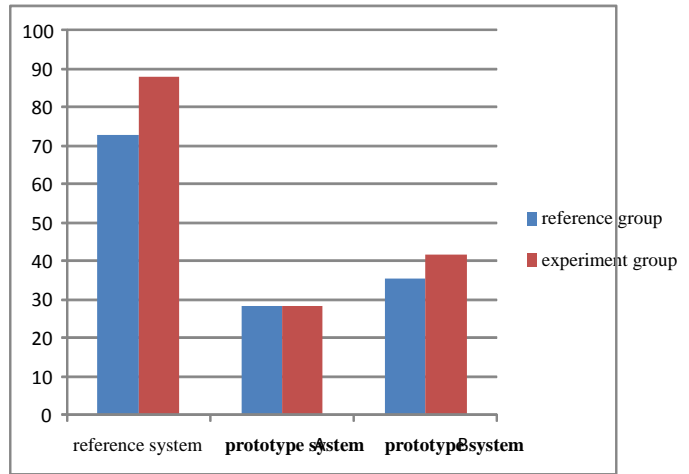

Fig.7. The comparison of the average values of reading completion time

The results through conducting the independent sample $T$ testing to the data acquired show that the users in the experiment group and those in the reference group have obviously differences in the satisfaction degrees, and have no significant differences in satisfaction degrees by using the experiment prototype system A and B. These prove that the reference system doesn't consider about the interactive experiences of the senile users, new users and users at low educational degrees, but the experiment prototype system fully considers about the user groups in need of care and doesn't influence the operation experiences of the mainstream user groups. Thus, the interface interactive design method with orientation of digital reading materials is effective to some degree.

\section{Conclusion}

The author takes the interface interactive design of digital reading material terminal as the case, explores the approach t establishing the interface interactive models and universal usable design matrix to reduce the feasibility of users' effort models, develops the prototype system in accordance with the proposed interface interactive design method, and verifies the validity of the method through control experiments. The experiment results prove that the interface interactive design method with the orientation of digital reading materials can improve users' operation performance and satisfaction, reduce the level of their effort models, and don't influence the operation experiences of the mainstream user groups while ensuring the interactive experiences of senile users, users with low educational degrees and new users. The interactive design method and design scheme are of instruction for the interface interactive design of the public information equipment.

\section{Acknowledgment}

Foundation program: Research on the users'experience of digital publication in new media era Wuhan Business and Technology University No.A2013019

Foundation program:Research on the users'experience of information interaction digital publication The humanities and social science program of the Education Department ofHubei Province No.14G 463 


\section{References}

[1] Alex Tek, Benoist Laurent, Marc Piuzzi, Zhihan Lu, Matthieu Chavent, Marc Baaden, Olivier Delalande et al. Advances in Human-Protein Interaction-Interactive and Immersive Molecular Simulations. InTech, 2012.

[2] Zhihan Lv, Alaa Halawani, Shengzhong Feng, Shafiq ur Rehman, Haibo Li. Touch-less Interactive Augmented Reality Game on Vision Based Wearable Device. Personal and Ubiquitous Computing. 2015.

[3] Shuping Dang, et al. Efficient solar power heating system based on lenticular condensation. 2014 International Conference on Information Science, Electronics and Electrical Engineering (ISEEE), 26-28 April 2014.

[4] Zhihan Lv, Liangbing Feng, Shengzhong Feng, and Haibo Li. Extending Touch-less Interaction on Vision Based Wearable Device. 2015 IEEE Virtual Reality (VR). IEEE, 2015.

[5] Yishuang Geng, J. He, H. Deng and K. Pahlavan, Modeling the Effect of Human Body on TOA Ranging for Indoor Human Tracking with Wrist Mounted Sensor, 16th International Symposium on Wireless Personal Multimedia Communications (WPMC), Atlantic City, NJ, Jun. 2013.

[6] Yishuang Geng, J. He, K. Pahlavan, Modeling the Effect of Human Body on TOA Based Indoor Human Tracking[J], International Journal of Wireless Information Networks 20(4), 306-317. 